NOTA TÉCNICA

\title{
FERTILIZACIÓN Y DENSIDAD DE POBLACIÓN EN LÍNEAS DE MAÍZ EN EL NORESTE DE MÉXICO1
}

\author{
Juan Antonio Morales ${ }^{2}$
}

\section{RESUMEN}

Fertilización y densidad de población en líneas de maíz en el noreste de México. Se estableció un ensayo de líneas de maíz (Zea mays L.) con diferentes niveles de N, P Y densidades de población. Se utilizó un diseño de parcelas divididas en bloques al azar en donde las líneas fueron la parcela principal mientras que la parcela pequeña fueron las combinaciones de N, P y densidades de población (para esta parcela se utilizó un diseño compuesto central para tres factores). Se presentó una diferencia significativa entre las líneas; no se presentó evidencia de interacción de los tratamientos con las líneas; un análisis posterior demostró que el punto estacionario era un punto silla. El análisis de cordillera demostró que la ruta de los máximos rendimientos seguía la tendencia $\mathrm{N}=83, \mathrm{P}=41$ y una densidad de población de 74.752 plantas por hectárea, lo cual no coincide con la recomendación que se hace al maíz comercial en esta región que es $\mathrm{N}=$ $140, \mathrm{P}=40$ y una densidad de 55.000 plantas por hectárea.

\begin{abstract}
Fertilization and population density in coro inbreds in Northeastern México. Three coro inbreds (Zea mays L.) with different levels of $\mathrm{N}, \mathrm{P}$ and population density were studied. The design used consisted of plots divided into randomized blocks where inbreds made up the main plot. Combinations of $\mathrm{N}, \mathrm{P}$ and population density made up the small plot (a three-factorial central composed design was used for in this plot). Results showed a significant difference between inbreds; there was no evidence of interaction between treatments and inbred combination. A subsequent analysis showed that the stationary point was a saddle point. Another analysis was carried out in order to determine this point, and it was found that the route to highest yields tended towards $\mathrm{N}=83, \mathrm{P}=41$, and a population density of 74,752 plants per hectare (pph). This contradicts the recommendation made for commercial coro in this region, which is $\mathrm{N}=140$, $\mathrm{P}=40$, and a population density of $55,000 \mathrm{pph}$.
\end{abstract}

\section{INTRODUCCIÓN}

En el noreste de México, hasta 1994, la región norte de Tamaulipas ha sobresalido por su importancia en el manejo y distribución de semillas para siembra, de tal forma que el $8 \%$ de la zona de riego (estimada en 310.000 hectáreas) se destinaba a la producción de semillas tanto de maíz como de sorgo y se disponía de potencial para la producción de semillas tanto para el consumo nacional como para la exportación. El CIRNoreste ha generado híbridos sobresalientes como el H422, H-435 y H-436; sin embargo, debido a la falta de presupuesto, el manejo agronómico de las líneas progenitoras de estos híbridos ha recibido atención limitada, pues para el manejo de un lote de producción de semillas se hacen las mismas recomendaciones que para la siembra de maíces comerciales, aún cuando el comportamiento de las líneas por sí mismas es diferente al del híbrido resultante de las cruzas de éstas.

La endogamia en maíz da por resultado un notable descenso del vigor y la productividad (Jugenheimer, 1976), los cuales se recuperan cuando se hace el cruzamiento de líneas no emparentadas. Además de esta pérdida, las plantas individuales de las primeras generaciones autofecundadas muestran muchos defectos, como reducción en altura, tendencia a producir chupones, acame, susceptibilidad a enfermedades y gran variedad de otras características desfavorables (Poehlman, 1979).

Entre los factores que reducen el riesgo del cultivo en la producción de semillas están la siembra de poblaciones que sean adecuadas (pero no tan densas

1 Trabajo financiado por el Programa Cooperativo Regional de Frijol de Centroamérica, México y el Caribe.

2 Campo Experimental Río Bravo. Apdo. Postal 70, Progreso, TX 78579 USA. Tel. (893)4-10-45. Fax. 4-60-20. Email: tlaloc2Ría>hotm,.il rom 
como para incrementar el riego por sequía) y el uso de un programa balanceado de fertilizantes, tanto iniciales como de distribución (Airy, mencionado por Jugenheimer, 1976).

La finalidad de la certificación es mantener y poner a la disposición del público semillas de alta calidad y propagación de plantas agrícolas superiores cultivadas y distribuidas de modo que se aseguren su identidad y pureza genética. En Estados Unidos, la Asociación de Agencias Oficiales Certificadoras de Semillas (AOSCA, por sus siglas en inglés) menciona que entre los requisitos para la elegibilidad de las variedades está el de una exposición de los planes y procedimientos para el mantenimiento de las clases de semilla básica (Jugenheimer, 1976).

En un estudio llevado a cabo con líneas de maíz se encontró que el calentamiento del suelo (el cual se hizo con cables eléctricos colocados bajo los surcos) tuvo un efecto marcado en las primeras etapas de desarrollo y en la absorción de $\mathrm{K}, \mathrm{P}$ y $\mathrm{Ca}$; además dicho calentamiento tendió a acortar el tiempo del período de emergencia a la etapa de floración femenina y no todas las líneas reaccionan en forma similar a los cambios de temperatura en el suelo (Jones y Medereski, 1963).

La producción de semillas justifica niveles de fertilidad óptima con el fin de maximizar (el uso) del agua de lluvia, irrigación, luz solar y las unidades calor (Craig, 1977); las cruzas simples o dobles pueden responder más que las líneas a las dosis altas de potasio (Gallaher et al., 1972).

Muchos investigadores han estudiado los efectos de las densidades en el rendimiento de maíz; sin embargo hay pocos estudios disponibles acerca de la respuesta de las líneas a las densidades de población; el efecto creciente de la contaminación de las aguas subterráneas sugiere la necesidad de una investigación más precisa de las recomendaciones de fertilizante (Wych, 1988). Tan importante como la correcta aplicación de las técnicas agrícolas para incrementar los rendimientos de un cultivo, es el aprovechamiento de los recursos con que se cuenta (Martínez, 1988).

El desarrollo de líneas endocriadas de maíz todavía consume la mayor parte de los programas de mejoramiento práctico. Por muchos años, cuando el producto que se iba a usar era el híbrido de cruza doble, posiblemente el mayor énfasis en el desarrollo de líneas fue su comportamiento en el híbrido; sin embargo, ahora, con un mayor uso de los híbridos de cruza simple, se ha incrementado el énfasis en el desarrollo de líneas que produzcan altas cantidades de semilla y de buena calidad (EI-Lakany y Rusel, 1971).

No obstante que la producción de semillas se considera una agroindustria importante, en el manual de producción de semilla publicado por el Patronato para la Investigación y Fomento de la Sanidad Vegetal (PIFSV) no se hace mención alguna acerca del manejo de las líneas progenitoras de maíz en un lote de producción de semillas en lo que se refiere a factores controlables tales como nitrógeno, fósforo, densidades de población, etc.

\section{MATERIALES Y MÉTODOS}

El experimento se estableció en el Campo Experimental Río Bravo, (CERIB), ubicado en Tamaulipas; se utilizaron las líneas $\mathrm{T}_{38}, \mathrm{~T}_{41} \mathrm{y} \mathrm{T}_{42}$, las cuales son las líneas progenitoras de los híbridos $\mathrm{H}-435$ y H-436; el diseño experimental fue el de bloques al azar en parcelas divididas con seis repeticiones; la parcela principal fueron las líneas, mientras que la parcela menor fueron las combinaciones de tratamientos de fertilización y densidades de población. El modelo lineal utilizado fue (Montgomery, 1984)

$y_{i j k}=\mu+\beta_{i}+v_{j}+(\beta v)_{i j}+-\tau_{k}+(\beta \tau)_{i k}+(v \tau)_{j k}+(\beta v \tau)_{i j k}$

en donde $y_{i j k}$ es la característica en estudio; $\beta_{i}$ es el efecto del bloque $i ; v_{j}$ es el efecto de la línea $j ;(\beta v)_{i j}$ es el efecto de interacción del bloque $i$ con la línea); $\tau_{k}$ es el efecto del tratamiento $k ;(\beta \tau)_{i k}$ es la interacción del bloque $i$ con el tratamiento $k ;(v \tau)_{j k}$ es el efecto de interacción de la línea) y el tratamiento $k$; finalmente $(\beta v \tau)_{i j k}$ es el efecto de la interacción del bloque $i$ con línea $k$ y el tratamiento $k$. En el Cuadro 1 se presenta la

Cuadro 1. Análisis de varianza.

\begin{tabular}{ll}
\hline \multicolumn{1}{c}{$\begin{array}{c}\text { Fuentes de } \\
\text { variación }\end{array}$} & \multicolumn{1}{c}{$\begin{array}{c}\text { Grados de } \\
\text { libertad }\end{array}$} \\
\hline Repeticiones (R) & $\mathrm{r}-1$ \\
Líneas (L) & $1-1$ \\
R X L & $(\mathrm{r}-1)(1-1)$ \\
Tratamientos (T) & $\mathrm{t}-1$ \\
Regresión & $\mathrm{q}$ \\
Falta de ajuste & $\mathrm{t}-1-\mathrm{q}$ \\
R X T & $(\mathrm{r}-1)(\mathrm{t}-1)$ \\
L X T & $(\mathrm{l}-1)(\mathrm{t}-1)$ \\
R X L X T & $(\mathrm{r}-1)(1-1)(\mathrm{t}-1)$ \\
Error de muestreo & $(\mathrm{m}-1) \mathrm{rlt}$ \\
\hline
\end{tabular}


partición de los grados de libertad. Las líneas se prueban contra la interacción R X L, mientras que los tratamientos de fertilización y densidades se probaron contra la interacción R X T; finalmente la interacción líneas $\mathrm{X}$ tratamientos $(\mathrm{L} \mathrm{X} \mathrm{T)}$ se probó contra la interacción R X L X T. En los tratamientos se utilizó un diseño compuesto central para tres factores con rangos de exploración, para $\mathrm{N}$ de 0 a $184 \mathrm{~kg} / \mathrm{ha}$; para $\mathrm{P}$ de 0 a $92 \mathrm{~kg} / \mathrm{ha}$; y para densidades de población 25.000 a $75.000 \mathrm{pph}$. Las dimensiones de la unidad experimental fueron de cuatro surcos de cinco metros de longitud y $0,80 \mathrm{~m}$ de ancho, en donde los datos se registraron en los dos surcos centrales, cada uno en forma independiente, esto es, cada UE constó de dos muestras; las características de porcentajes de grano y humedad sólo se registraron en tres repeticiones. El experimento se sembró el 25 de febrero de 1994 y se cosechó el 26 de julio de ese mismo año.

\section{RESULTADOS}

\section{Análisis de la parcela mayor (líneas)}

En el Cuadro 2 se presenta un resumen de las características registradas así como su significancia; nótese que ninguna característica presentó interacción entre las líneas y los tratamientos. La separación de los promedios se realizó por el método de Tukey $(\alpha=0,05)$ se presenta en el Cuadro 3. Puede apreciarse que la línea de mayor rendimiento fue la T38; el material que presentó menor porcentaje de humedad a cosecha fue la línea $\mathrm{T}_{41}$, al mismo tiempo que también presentó un mayor porcentaje de grano; en cuanto a la altura de planta, la $T_{38}$ y la $T_{42}$ presentaron, en forma significativa, una altura mayor que la $\mathrm{T}_{41}$, mientras que el mayor porcentaje de mazorcas buenas correspondió a la $\mathrm{T}_{42}$.

\section{Análisis en la parcela menor (combinacines de tratamientos de fertilización y densidades)}

Para la característica rendimiento se procedió a particionar la suma de cuadrados para tratamientos como se indica en el Cuadro 4. El modelo de regresión ajustado fue:

$$
\begin{gathered}
y=286.751-3.311 N+10.008 P+78.107 D+0.021 \\
N^{2}-0.003 P N-0.0369 P^{2}-0.0128 D N-0.115 D P- \\
0.257 D^{2}
\end{gathered}
$$

en donde $\mathrm{N}$ es nitrógeno $(\mathrm{kg} / \mathrm{ha}), \mathrm{P}$ es fósforo ( $\mathrm{kg} / \mathrm{ha}$ ) y D es la densidad (plantas por hectárea). Puede apreciarse que la falta de ajuste es no significativa, lo cual sugiere que el. modelo propuesto se ajusta bien a los datos.

Cuadro 2. Resultados del análisis de varianza para rendimiento y otras características agronómicas de líneas progenitoras de maíz.

\begin{tabular}{lccccc}
\hline \multirow{2}{*}{$\begin{array}{l}\text { Fuente de } \\
\text { variación }\end{array}$} & rendimiento & humedad & \multicolumn{2}{c}{ Altura de } & mazorcas \\
\cline { 4 - 5 } & & $(\boldsymbol{\%})$ & planta & mazorca & buenas (\%) \\
\hline Líneas & $* *$ & $* *$ & $* *$ & $* *$ & $* *$ \\
Tratamientos & $* *$ & $\mathrm{~ns}$ & $* *$ & $\mathrm{~ns}$ & $* *$ \\
Interacción & $\mathrm{ns}$ & $\mathrm{ns}$ & $\mathrm{ns}$ & $\mathrm{ns}$ & $\mathrm{ns}$ \\
\hline
\end{tabular}

**Significativo al $0.01 \%$ de probabilidad.

Cuadro 3. Promedios* de rendimiento y otras características agronómicas de líneas pogenitoras de maíz.

\begin{tabular}{lcccccc}
\hline Líneas & $\begin{array}{c}\text { Rendimiento } \\
(\mathbf{k g} / \mathbf{h a})\end{array}$ & $\begin{array}{c}\text { Humedad } \\
(\%)\end{array}$ & $\begin{array}{c}\text { Grano } \\
(\boldsymbol{\%})\end{array}$ & $\begin{array}{c}\text { Altura } \\
\text { planta }\end{array}$ & $\begin{array}{c}\text { Cm de } \\
\text { mazorca }\end{array}$ & $\begin{array}{c}\text { Mazorcas } \\
\text { buenas (\%) }\end{array}$ \\
\hline $\mathrm{T}_{38}$ & $4336 \mathrm{a}$ & $13,05 \mathrm{a}$ & $80,67 \mathrm{~b}$ & $158 \mathrm{a}$ & $51 \mathrm{~b}$ & $80 \mathrm{~b}$ \\
$\mathrm{~T}_{41}$ & $3499 \mathrm{~b}$ & $12,81 \mathrm{~b}$ & $82,82 \mathrm{a}$ & $138 \mathrm{~b}$ & $41 \mathrm{c}$ & $82 \mathrm{~b}$ \\
$\mathrm{~T}_{42}$ & $2670 \mathrm{c}$ & $14,09 \mathrm{a}$ & $75,06 \mathrm{c}$ & $155 \mathrm{a}$ & $58 \mathrm{a}$ & $90 \mathrm{a}$ \\
\hline
\end{tabular}


En el Cuadro 5 se presentan otras características registradas; de este cuadro puede mencionarse que la $\mathrm{T}_{42}$ fue la línea que presentó el mayor número de días a floración masculina y tuvo el menor porcentaje de mazorcas con mala cobertura; la línea T41 presentó el menor porcentaje de plantas acamadas; tanto el porcentaje de Ustilago maydis como el de plantas sin mazorca pueden considerarse irrelevantes; nótese también que la $\mathrm{T}_{42}$ presentó un porcentaje de cuateo bastante alto $(24,91 \%)$ en comparación con las otras líneas.
Cuadro 4. Partición de la suma de cuadrados para tratamientos.

\begin{tabular}{lcrc}
\hline $\begin{array}{l}\text { Fuente de } \\
\text { variación }\end{array}$ & $\begin{array}{c}\text { Grado de } \\
\text { libertad }\end{array}$ & $\begin{array}{c}\text { Cuadrados } \\
\text { medios }\end{array}$ & Fo \\
\hline Tratamientos & 14 & & \\
Regresión & 9 & 21677323.8 & \\
Falta de ajuste & 5 & 735347.5 & 2.82 \\
Error & 70 & 260736.0 & \\
\hline
\end{tabular}

Cuadro 5. Otras características registradas.

\begin{tabular}{lccrcrc}
\hline & & \multicolumn{5}{c}{ Porcentaje de plantas } \\
\cline { 3 - 6 } Línea & $\begin{array}{c}\text { Días a } \\
\text { floración }\end{array}$ & $\begin{array}{c}\text { Mala } \\
\text { cobertura }\end{array}$ & Acame & $\begin{array}{c}\text { Ustilago } \\
\text { Maydis }\end{array}$ & Cuateo & Sin mazorca \\
\hline $\mathrm{T}_{38}$ & 75 & 18.40 & 16.00 & 3.23 & 1.59 & 1.85 \\
$\mathrm{~T}_{41}$ & 75 & 20.42 & 9.85 & 4.14 & 1.34 & 2.50 \\
$\mathrm{~T}_{42}$ & 83 & 14.94 & 11.70 & 3.47 & 24.91 & 1.76 \\
\hline
\end{tabular}

\section{DISCUSIÓN}

En la actualidad no se tiene una recomendación agronómica exclusiva para el manejo de las líneas de maíz en los lotes para la producción de semilla; la sugerencia que se hace es la de aplicar las recomendaciones para el maíz comercial, a saber 140 $\mathrm{kg} / \mathrm{ha}$ de $\mathrm{N}, 40 \mathrm{~kg} / \mathrm{ha}$.de $\mathrm{P}$ y $55.000 \mathrm{pph}$; cuando se solicita la caracterización de alguna línea determinada, ésta se limita a datos como la altura de planta, mazorca, fecha de siembra, etcétera, que si bien son importantes, no dan información acerca del manejo que se debería llevar a cabo en el lote de producción de semilla. Al realizar el análisis canónico, se encontró que el punto estacionario era un punto silla; al seguir la ruta de los rendimientos máximos (para 10 cual se realizó un análisis de cordillera (ver Martínez-Garza, 1988), se obtuvo $\mathrm{N}=83, \mathrm{P}=41$ y con una densidad de población de $74.752 \mathrm{pph}$, es decir, para el nitrógeno se puede sugerir aplicar una dosis de 83 unidades por hectárea, para fósforo, 41 unidades por hectárea y finalmente, para la población de plantas, se sugieren $74.752 \mathrm{pph}$. Las líneas típicamente producen menos biomasa y rendimiento que el híbrido resultante; la emasculación del progenitor femenino y la posterior destrucción del progenitor masculino después del derramamiento del polen puede también tener un efecto enlos requerimientos de nitrógeno para la producción de semilla (Peterson y Corak, 1993). Debe recordarse que los niveles de humedad del suelo necesitan mantenerse en equilibrio puesto que las líneas son más vulnerables a las deficiencias nutricionales que los híbridos debido a las diferencias genéticas y a la capacidad de enraizamiento (Craig, 1977) además de que difieren en su capacidad para absorber hierro y zinc (Kamprath y Foy, mencionados por Larson y Hanway, 1977); las cruzas simples o dobles pueden responder más que las líneas a las dosis de potasio (Gallaher et al., 1972).

El presente estudio es un intento por determinar los efectos de los factores controlables de producción (en este caso $\mathrm{N}, \mathrm{P}$ y densidades de población) en el rendimiento, calidad y otras características agronómicas en las líneas progenitoras de híbridos de maíz generados en este instituto. Se sugiere que en los próximos estudios se establezcan más localidades (tentativamente 10), con el fin de estudiar las posibles interacciones de estos factores y los diferentes tipos de suelo.

\section{CONCLUSIONES}

1. La línea $T_{38}$ fue superior al resto de los materiales en lo que se refiere a rendimiento.

2. En el presente estudio se encontró que tanto las dosis para $\mathrm{N}$ y $\mathrm{P}$ como la densidad de población no coinciden con las recomendaciones hechas para el maíz comercial.

3. El porcentaje de humedad a cosecha fue menor para la línea $\mathrm{T}_{41}$. 
4. El porcentaje de grano fue menor en la línea $\mathrm{T}_{41}$.

5. Las líneas $\mathrm{T}_{38}$ y $\mathrm{T}_{42}$ fueron las líneas que presentaron mayor altura de planta, mientras que la $\mathrm{T}_{41}$ fue la de menor altura.

6. La línea $\mathrm{T}_{42}$ fue el material que presentó mayor porcentaje de mazorcas buenas

7. En las próximas etapas se sugiere establecer un número mayor de localidades (tentativamente diez) y así determinar las posibles interacciones de estos factores y los diferentes tipos de suelo y/o localidad.

\section{LITERATURA CITADA}

CRAIG, W. F. 1977. Production of hybrid com seed. In G. F. Sprague (editor). Corn and corn improvement. American Society of Agronomy, Inc., Publishier. Madison, Winsconsin.

EL-LAKANY, M. A.; RUSELL, W. A. 1971. Relationship of maize characters with yield in testcrosses at different plant densities. Crop Science 11: 698 - 701.

GALLAHER, R. N.; PARKS, W. L.; JOSEPHSON, L. M. 1972. Effects of levels of soil potassium, fertilizer potassium, and season on yield and leaf potassium content of corn inbreds and hybrids. Agronomy Journal 64: 645-647.
JUGENHEIMER, R. W. 1976. Corn: improvment, seed production and uses. John Wiley \& Sons, Inc.

JONES JR., J. B.; MEDERESKI, H. J. 1963. Effect of soil temperature on corn plant development and yield: II. Studies with six inbred lines. Soil Science Proceedings 27: 189-192.

LARSON, W. E.; HANWAY, J. J. 1977. Corn production. In: G. F. Sprague (editor). Corn and corn improvement. ASA, Inc. Publishier. Madison, Winsconsin.

MARTÍNEZ GARZA, A. 1988. Diseños experimentales. Editorial Trillas, México. p. 336, 363.

MONTGOMERY, D. C. 1984. Design and analysis of experi ments.Segunda edición. John Wiley \& Sons. p. 386.

PETERSON, R.; CORAK, S. 1993. Nitrogen response in seed corn production. Proceedings of the forty-eighth annual corn \& sorghum industry research conference: 169-189.

POEHLMAN, J. M. 1979. Breeding field crops. $2^{\text {da }}$ edición.The AVI Publishing Company, Inc.

WYCH, R. D. 1988. Production of hybrid seed corn. In G. F. Sprague y J. W. Dudley (editores). Corn and corn improvement. American Society of Agronomy, Ing. Publisher. Madison, Wisconsin, USA. 